\title{
Beaucoup d'initiatives conduisent au budget global
}

\section{Bruno Henggi}

Responsable Affaires publiques de la FMH

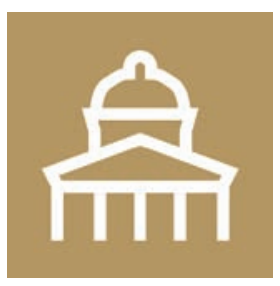

Fin juin, le président du PDC a annoncé au SonntagsBlick que son parti avait déjà récolté plus de la moitié des 100000 signatures pour le frein aux coûts de la santé. De son côté, l'institut de recherche gfs a également rappelé dans le Moniteur de la santé de cette année que, pour l'heure, les électeurs sont d'accord sur le fond avec cette initiative [1]. Tout d'abord, le parti mérite d'être félicité, mais force est de constater que ces résultats ne sont pas très surprenants. Vouloir baisser les primes est un postulat contre lequel il est difficile de s'opposer. En revanche, il serait intéressant de demander aux personnes qui signent si elles croient réellement aux promesses de l'initiative. Le même Moniteur de la santé gfs fait remarquer que les électeurs s'attendent encore clairement à ce que les coûts de la santé et les primes d'assurance-maladie augmentent [2].

Un des atouts de l'initiative est sans aucun doute de ne pas se confronter aux bassesses du débat sur l'impact et les effets indésirables de mesures spécifiques. Si l'initiative est acceptée, le Conseil fédéral et le Parlement devront fournir les pistes pour mettre en œuvre le frein aux coûts. Le texte de l'initiative ne donne aucune indication à ce sujet. Les initiants sont suffisamment prudents pour éviter les points d'achoppement et ne nommer aucune mesure concrète. En d'autres termes, on ne souhaite pas être associé au budget global.

C'est tout à fait compréhensible: dans le Moniteur de la santé gfs de l'année dernière, 91\% des personnes interrogées associaient le budget global à des temps d'attente plus longs et s'attendaient à une limitation du libre choix du médecin; $86 \%$ validaient la déclaration selon laquelle le budget global péjorait la qualité des soins et, enfin, la majorité (54\%) pensait que le budget global n'aurait pas l'effet d'un frein sur les primes [3]. Le budget global est une notion que le Département de l'intérieur (DFI) n’apprécie pas (plus). Il préfère désormais parler d'objectifs de maîtrise des coûts de la santé, qui seraient atteints au travers par exemple de budgets globaux pour le domaine ambulatoire ou de tarifs dégressifs. Ce dernier instrument devrait figurer au point
Pilotage des coûts par les partenaires tarifaires du message du DFI sur le premier volet de mesures visant à maîtriser les coûts, qui sera remis au Parlement à la rentrée [4]. En agissant ainsi, le département cible la compétence subsidiaire qui lui permettra d'imposer les tarifs dégressifs et les baisses de tarifs. Enfin, l'ouverture de la consultation sur le pilotage global de l'assurance obligatoire via des plafonds contraignants (ou pas) a été annoncée pour la fin de l'année [5].

Les assurés, les patients et les payeurs de primes suivront avec intérêt les discussions relatives à ces projets. Car c'est le principe d'assurance, inscrit dans la Constitution fédérale, qui est en jeu. Ce principe stipule qu'une prestation assurée par contrat doit être fournie en cas de risque. Tout assuré voudra donc savoir si et dans quelle mesure une limitation du volume des prestations engendrée directement par le budget global impactera la prestation promise pour son cas particulier. Le patient s'intéressera aux conséquences que pourrait avoir une tarification insuffisante sur les prestations de l'assurance obligatoire. Devra-t-il contracter une assurance complémentaire pour se prémunir des temps d'attente ou, au cas par cas, s'adresser à un autre médecin? Il se demandera avec inquiétude si, en tant que patient de l'assurance obligatoire, il pourra continuer de bénéficier de ce qui est médicalement nécessaire ou s'il devra se contenter de ce qui est économiquement possible.

\section{Références}

1 Bieri Urs, et al. La qualité avant les coûts, moins de disposition à renoncer, L'essentiel en bref du Moniteur de la santé 2019, édité par Interpharma 2019, p. 22.

2 Bieri Urs, et al. La qualité avant les coûts, moins de disposition à renoncer, L'essentiel en bref du Moniteur de la santé 2019, édité par Interpharma 2019, p. 11

3 Bieri Urs, et al. Pas d'expériences souhaitées, mais plus d'exigences vis-à-vis de la prise en charge, L'essentiel en bref du Moniteur de la santé 2018, édité par Interpharma 2018, p. 8.

4 Office fédéral de la santé publique. Révision LAMal: $1^{\text {er }}$ volet de mesures visant à maîtriser les coûts. Consultation du 14 septembre 2018.

5 Office fédéral de la santé publique. Le Conseil fédéral examine un objectif de maîtrise des coûts de la santé. Communiqué de presse du 8 mars 2019. 